SMAD, Rev. Eletrônica Saúde Mental Álcool Drog.

2019 jul.-sept.;15(3):1-6

DOI: 10.11606/issn. 1806-6976.smad.2019.151296

www.revistas.usp.br/smad/

Artículo Original

\title{
Modelo bioecológico para el autocontrol del consumo de alcohol y calidad de vida laboral
}

\section{Alejandra Leija Mendoza ${ }^{1}$ \\ (iD) https://orcid.org/0000-0001-6806-8915 \\ Alicia Álvarez Aguirre ${ }^{1}$ \\ (D) https://orcid.org/0000-0001-5538-7634 \\ Ana Carolina Guidorizzi Zanetti2 \\ (D) https://orcid.org/0000-0003-0011-4510}

Objetivo: el propósito del estudio fue derivar "The Ecology of Human Development" para explicar el autocontrol del consumo de alcohol y calidad de vida en el trabajo desde el enfoque de enfermería. Método: análisis y aplicación de la metodología la cual contempla cinco pasos principales. Resultados: construcción del modelo Bioecológico para el autocontrol del consumo de alcohol y calidad de vida en el área laboral. Conclusiones: el desarrollo del modelo puede favorecer identificar y generar campos de acción que sean útiles en la práctica del enfermero con fundamento científico para prevenir o disminuir el consumo de alcohol en el área laboral.

Descriptores: Trabajadores; Teoría Bioecológica; Derivación de Teoría; Enfermería.

\section{Cómo citar este artículo}

Mendoza AL, Aguirre AA, Zanetti ACG. Bioecological model for the self-control of alcohol consumption and quality of work life. SMAD, Rev Eletrônica Saúde Mental Álcool Drog. 2019;15(3):1-6. doi: https://dx.doi.org/10.11606/issn.1806-6976.smad.2019.151296 


\title{
Bioecological model for the self-control of alcohol consumption and quality of work life
}

\begin{abstract}
Objective: the purpose of the study was to derive "The Ecology of Human Development" to explain the self-control of alcohol consumption and quality of life at work from the nursing approach. Method: analysis and application of the methodology which includes five main steps. Results: bioecological model for self-control of alcohol consumption and quality of life in the workplace. Conclusions: the development of the model can favor identifying and generating fields of action that are useful in the practice of nurses with a scientific basis to prevent or reduce the consumption of alcohol in the workplace.
\end{abstract}

Descriptors: Workers; Bioecological Theory; Derivation; Nursing.

\section{Modelo bioecológico para o autocontrole do consumo de álcool e qualidade de vida no trabalho}

\begin{abstract}
Objetivo: a proposta do estudo foi derivar a "The Ecology of Human Development" para explicar o autocontrole do consumo de álcool e a qualidade de vida no trabalho a partir da abordagem da enfermagem. Método: análise e aplicação da metodologia que inclui cinco etapas principais. Resultados: construção do modelo bioecológico para autocontrole do consumo de álcool e qualidade de vida no local de trabalho. Conclusões: o desenvolvimento do modelo pode favorecer a identificação e geração de campos de ação que sejam úteis na prática dos enfermeiros com base científica para prevenir ou reduzir o consumo de álcool no local de trabalho.
\end{abstract}

Descritores: Trabalhadores; Teoria Bioecológica; Derivação; Enfermagem. 


\section{Introducción}

La mayor proporción de enfermedad y de años perdidos a causa del consumo de alcohol ocurre en América juntamente con los problemas sociales, mentales y emocionales afectando no sólo al consumidor si no a las personas a su alrededor ${ }^{(1)}$, en México la prevalencia del consumo de alcohol en el último mes es de $31.6 \%$ en población con edad entre 12 y 65 años, siendo la cerveza la bebida de preferencia. El panorama actual hace con que la población en general sea susceptible a presentar este tipo de conductas a edades más tempranas al reportar la ENCODAT por personas que consumieron alcohol por primera vez a los 17 años ${ }^{(2)}$.

El ambiente laboral no es una excepción, la Organización Internacional del Trabajo informa que el consumo de alcohol representa una conducta de riesgo con altas prevalencias debido a la aceptación social y cultural provocando condiciones de riesgo dentro y fuera de su área laboral(3-4). Así mismo la normalidad de consumo en este ambiente puede deberse a las altas demandas de trabajo, realizar tareas rutinarias, manuales y monótonas, insatisfacción laboral, jornadas de trabajo prolongadas, estrés, rotación de turnos constantes, cansancio, tener compañeros consumidores y en algunos de los casos la accesibilidad de alcohol en el trabajo(5).

Hay evidencias de una normalidad de consumo riesgoso en los trabajadores quienes tienen la posibilidad de presentar accidentes y lesiones laborales, las áreas comunes afectadas por esto son dedos, brazos, piernas y ojos, ya que el alcohol afecta la percepción, el equilibrio y la coordinación haciendo con que la productividad y la calidad con la que realiza sus tareas laborales disminuya afectando la Calidad de Vida Laboral (CVL) ${ }^{(6-10)}$.

La CVL es un concepto multidimensional donde el trabajador ve una necesidad no atendida en relación a diversos factores como soporte institucional, seguridad en el trabajo, organización laboral, el bienestar, condiciones de trabajo e incluso aspecto físico y mental del trabajador, por lo que la valoración de estos aspectos juntamente con estilos de vida o comportamientos de un empleado permitirán realizar acciones oportunas que promuevan la $\mathrm{CVL}^{(11)}$.

En este sentido, algunas teorías permiten explicar el conocimiento de fenómenos amplios y complejos con características en diferentes niveles relacionados a la prevención y el cuidado. "La Ecología de Desarrollo Humano" propone un modelo que le permite a la enfermería determinar el desarrollo de conductas como resultado de la interacción de una persona con configuraciones diferentes ${ }^{(12-13)}$.

Esta teoría determina que el desarrollo es el producto de la interacción activa entre la persona (trabajador) y su ambiente, del cual se pueden obtener conductas y cambios de las mismas a partir del contexto social y económico en el que se desenvuelve, una vez que la conducta emerge a partir del intercambio que existe entre la persona con el ambiente. A su vez, él explica que dicho desarrollo se da a partir de tres características el individuo que es capaz de reestructurarse (disminución del consumo de alcohol), la reciprocidad entre el ambiente y el sujeto y el ambiente que es representado por varios ambientes interconectados que emanan de la persona (laboral, familiar y social) y que a su vez son capaces de explicar o predecir el desarrollo de conductas nocivas como el consumo de alcohol.

Esa teoría proporciona un fundamento teórico amplio mediante el cual los profesionales de enfermería puedan desarrollar programas e intervenciones para trabajadores consumidores de alcohol, para detectar como algunos de los ambientes en los que se desarrolla un trabajador influyen como factores protectores para tener autocontrol sobre el consumo de alcohol.

La metodología adecuada para desarrollar teoría en la que se emplee un nuevo enfoque es la derivación teórica, la cual es útil cuando se carecen de teorías y modelos que abordan fenómenos complejos de estudio que muestran relaciones entre los diversos conceptos. En este sentido, la derivación proporciona estructuras y relaciones sobre un fenómeno o conducta determinada con base en evidencias existentes. Es por lo tanto que el propósito del presente artículo es presentar la derivación teórica y fundamentación científica "The Ecology of Human Development"(12).

\section{Método}

Para realizar la derivación teórica se utilizó la siguiente propuesta: 1) Revisión de la literatura relacionada al fenómeno de estudio, 2) Identificar los conceptos, definiciones y relaciones principales desde una perspectiva filosófica, 3) Identificación del modelo, 4) Desarrollo de las propuestas y proposiciones con base a la estructura de las preposiciones de la teoría seleccionada y en la literatura seleccionada del fenómeno de estudio y 5) Construcción del nuevo modelo redefiniendo las proposiciones derivadas y adaptadas del fenómeno de estudio(14).

\section{Resultados y Discusión}

En el primer paso se realizó una revisión de la literatura de la teoría de interés, el consumo de alcohol en el área laboral, así como los factores de protección o consumo de alcohol en riesgo para el trabajador. La búsqueda se realizó con bases de datos Scopus, Ebsco, Proquest, Medline, Science Direct, Scielo; las palabras claves utilizadas fueron: uso de alcohol, trabajadores y calidad de vida.

En el segundo paso se identificó el fenómeno de estudio desde diferentes perspectivas (psiquiatría, psicología, medicina, enfermería, salud mental, ergonomía, seguridad y salud en el trabajo, drogas y dependencia de alcohol), se analizó la temática de los estudios; en primer lugar, desde las diferentes áreas laborales (industria, construcción, personal de salud, conductores, administración, docencia) con el objetivo de analizar las normalidades de consumo, así como los factores protectores o de riesgo para el uso de alcohol destacando el área industrial donde el consumo es frecuente y elevado. 
Se identificaron algunos de los conceptos básicos "La Ecología del Desarrollo Humano", la cual se explica como los diversos ambientes afectan en el cambio de una conducta específica, en este sentido se analizó la temática desde la perspectiva psicológica y social en las cuales también se identifica los contextos, y los diversos ambientes y condiciones que favorecen el consumo de alcohol en la población activa.

En el tercer paso se seleccionó "The Ecology of Human Development", con la que se realizó la derivación teórica conceptual empírica y se definió la estructura y las preposiciones. La selección del modelo se estableció con base al objeto, perspectiva y conceptos básicos; este modelo se utiliza para desarrollar intervenciones multinivel e integral a través de diversos ambientes que orienta cambios de conducta en el área de salud mediante la influencia que puede dar en cada uno de los niveles del modelo(12).

La "Ecología del Desarrollo Humano", en primer lugar, considerado en su paradigma del proceso, persona, ambiente y tiempo. El proceso se refiere que es el responsable del desarrollo mediante la interacción entre el individuo y las personas, objetos o símbolos de su entorno inmediato. La persona es quien posee factores biológicos, genéticos, características personales y atributos para el desarrollo. La atmósfera es la condición de la influencia externa e influye en el sujeto y está compuesto por cuatro subsistemas que sostienen y apoyan el desarrollo. El tiempo se refiere a las estructuras que puedan generar cambios en el ambiente o en la vida de las personas que enfrentan transiciones en su desarrollo(12).

El ambiente incluye cuatro niveles principales o estructuras de serie y cada una de ellas cabe dentro de la siguiente, estos niveles representan parte de las 11 definiciones básicas de su teoría, los niveles fijados son los siguientes: 1) microsistema, 2) mesosistema, 3) exosistema y 4) macrosistema(12).

El primer nivel llamado microsistema se define como "una normalidad de actividades, funciones y relaciones en que la persona en desarrollo experimenta en un entorno de determinado ambiente, con características físicas y materiales particulares". El segundo nivel nombrado mesosistema se refiere como "interrelaciones de dos o más entornos en los que la persona en desarrollo participa activamente"(12).

El tercer nivel nombrado exosistema se refiere a "uno o más entornos en los que no se incluye a la persona como participante activo", sin embrago los acontecimientos en este ambiente afectan la persona.
Por último, el macrosistema hace referencia a lo que corresponde en "forma y contenido de cada uno de los sistemas anteriormente mencionados a nivel de la cultura, creencias e ideologías, política, estilos de vida del individuo", ya que cada una de estas características pueden afectar el bienestar, el desarrollo humano y a su vez determinar las condiciones de vida de los sujetos ${ }^{(12)}$.

En el cuarto paso se desarrolló una nueva propuesta con base a la temática de estudio, las preposiciones, los conceptos de la teoría seleccionada y la literatura revisada del fenómeno de estudio en el área de enfermería, así como de otros campos disciplinares. La "Ecología del Desarrollo Humano," incluye un total de nueve preposiciones; la preposición enfocada al fenómeno de estudio indica que "los diferentes tipos de entorno dan lugar a distintas normalidades ejercidas, actividad y relación para las personas que se convierten participantes de estos entornos"(12), en este sentido la literatura señala que diversos ambientes intervienen en el consumo de alcohol del trabajador, uno de ellos se encuentra definido por el ambiente laboral el cual está determinado por las características físicas y materiales, así como la influencia de los compañeros de trabajo, otro que influye es el ambiente familiar que puede ser un factor de protección para el consumo de alcohol ya sea por la influencia que esta tiene sobre la persona o por el número de personas que componen el núcleo en el que se desenvuelve y por último el ambiente social en el que se refiere a las relaciones interpersonales que el trabajador puede representar un factor de protección para el uso de alcohol.

La teoría considera, una de nueve preposiciones, "los diferentes tipos de entorno dan lugar a patrones distintivos del rol, actividad y relación para que las personas se conviertan en participantes de estos entornos"(12). La evidencia muestra que las personas al adquirir el papel de trabajador en diversas condiciones y la influencia de las relaciones interpersonales familiares, laborales o sociales adquiridas pueden desarrollar conductas poco favorables para la salud como el consumo de alcohol y adquirir un uso frecuente en los fines de semana, tipo de jornada laboral o la demanda de la misma ${ }^{(5-12)}$. Lo anterior refiere que los entornos en los que se desenvuelve una persona definen los comportamientos que puede adquirir o cambiar bajo la influencia de los mismos, esto ha sido referido desde varias áreas disciplinares, así como por organizaciones internacionales como la OIT como una forma de influir en los entornos laborales mediante la promoción de la salud.

\begin{tabular}{|l|l|}
\hline $\begin{array}{l}\text { Proposiciones de la Teoría Bioecológica del Desarrollo } \\
\text { Humano }\end{array}$ & $\begin{array}{l}\text { Proposiciones del Modelo Bioecológico para el Autocontrol del Consumo } \\
\text { de Alcohol y CVL* }\end{array}$ \\
\hline $\begin{array}{l}\text { "Los diferentes tipos de entorno dan lugar a los patrones distintivos } \\
\text { del rol, actividad y relación para las personas que se convierten en } \\
\text { participantes de estos entornos". }\end{array}$ & $\begin{array}{l}\text { "Los ambientes laboral, familiar y social para el autocontrol del consumo de } \\
\text { alcohol y la CVL son interactivos entre si". }\end{array}$ \\
\cline { 2 - 3 } & $\begin{array}{l}\text { "Los ambientes laboral, familiar y social influyen de forma positiva en el } \\
\text { autocontrol del consumo de alcohol y la CVL". }\end{array}$ \\
\hline
\end{tabular}

*CLV - Calidad de Vida Laboral; Fuente: derivado "The Ecology of Human Development"(12)

Figura 1 - Preposiciones consideras por la Teoría Bioecológica del Desarrollo Humano y del Modelo Bioecológico para el Autocontrol del Consumo de Alcohol y CVL 
Por último, en el quinto paso se redefinieron los conceptos que fueron adaptados al fenómeno de estudio, de esta manera se adquirieron nuevos conceptos procedentes de la teoría madre y con base a la evidencia científica encontrada durante el proceso de revisión de la literatura

Ambiente Laboral: Ambiente en el que el trabajador desarrolla sus actividades por lo que es considerado su entorno inmediato el cual contiene características físicas y materiales particulares que son capaces de moldear el autocontrol del consumo de alcohol y la Calidad de Vida Laboral (CVL).
Ambiente Familiar: Es la forma en la que una persona percibe el funcionamiento de su familia en el autocontrol del consumo de alcohol.

Ambiente Social: Representa un elemento de la persona que puede regular problemas de salud y psicológicos.

El ambiente familiar y el Ambiente social son permeables y bidireccionales con el ambiente laboral del trabajador al ser un participante activo múltiple al contemplar la interrelación de dos ambientes que influyen para que el trabajador desarrolle el autocontrol del consumo de alcohol y la CVL.

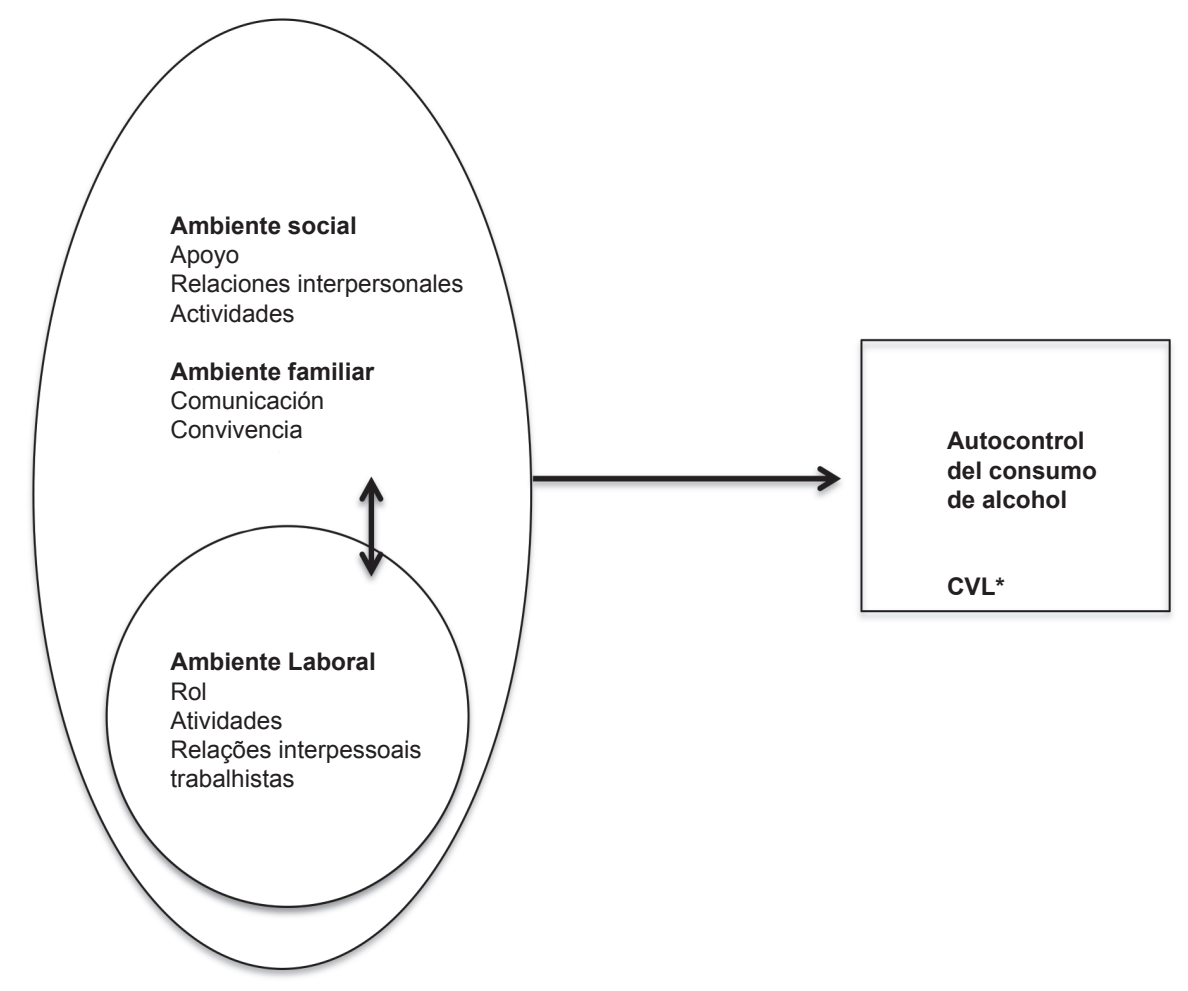

*CLV $=$ Calidad de Vida Labora

Figura 2 - Adaptación de la Teoría Bioecológica del Desarrollo Humano para el Autocontrol del Consumo de Alcohol y la Calidad de Vida Laboral (CVL)

En la Figura 2 se muestra la adaptación del Modelo Bioecológico para el Autocontrol del Consumo de Alcohol y la Calidad de Vida Laboral (CVL)

\section{Conclusiones}

El desarrollo del modelo promueve en el profesional de enfermería la interacción en el área laboral con el objetivo de prevenir o disminuir el consumo de alcohol, al ser este un fenómeno de alto impacto reconocido por organizaciones internacionales en el que si bien es cierto ha sido abordado por diferentes áreas disciplinares, enfermería carece de un papel proactivo en el área laboral.

Es por esto que enfermería debe de realizar actividades dirigidas a la promoción, prevención, preservación y recuperación de la salud en la población trabajadora con base científica en el cuidado integral mediante un enfoque laboral en la prevención de riesgos procedentes de las diversas áreas laborales específicas y de los comportamientos adquiridos que representan un riesgo de salud para el trabajador.

Lo anterior se puede lograr mediante el desarrollo y la incorporación de teorías y modelos, siendo esto parte de un crecimiento científico y fundamentado de enfermería capaz de guiar cada una de las actividades independientes que enfermería pude realizar y generar líneas de acción específicas para problemáticas con altas prevalencias e impacto social como el consumo de alcohol.

En este sentido, la metodología representa una herramienta útil que permite contextualizar fenómenos que por su naturaleza y complejidad no se pueden delimitar de forma precisa; es por esto que el Modelo 
Bioecológico para el Autocontrol del Consumo de Alcohol y CVL permite observar al fenómeno en sus diferentes niveles de interacción y explicar cómo el trabajador puede adquirir y cambiar conductas que representan un riesgo para su salud a través de los diversos factores o características que propician el uso de alcohol, las cuales se pueden identificar y generar campos de acción que sean útiles en la práctica del enfermero con fundamento científico para prevenir o disminuir el consumo de alcohol en el área laboral.

\section{Referencias}

1. Anderson P. Gual A. Colon J. Alcohol y atención primaria de la salud: informaciones clínicas básicas para la identificación y el manejo de riesgos y problemas. [Internet]. Washington: D.C. OPS; 2008 [Acceso 7 feb 2016]. Disponible en: http://www.who.int/substance_ abuse/publications/alcohol_atencion_primaria.pdf

2. Instituto Nacional de Psiquiatría Ramón de la Fuente Muñiz; Instituto Nacional de Salud Pública, Comisión Nacional Contra las Adicciones, Secretaria de Salud. Encuesta Nacional del Consumo de Drogas, Alcohol y Tabaco 2016-2017 (ENCODAT): Reporte de Alcohol. [Internet]. Ciudad de México, México: INPRFM; 2017. [Acceso 12 marzo 2018]. Disponible en: www.conadic. gob.mx

3. International Labour Organization. Prevention of alcohol and drugs use in the workplace: A consensual approach to workplace substance abuse: from rehabilitation to prevention [Internet]. Ginebra; 2016. [Acceso 5 feb 2016]. Disponible en: http://www.ilo.org/ safework/areasofwork/workplace-health-promotion-andwell-being/WCMS_108398/lang--en/index.htm

4. Organización Internacional del Trabajo. Misión e Impacto de la OIT: promover el empleo, proteger a las personas. [Internet]. Ginebra; 2016. [Acceso 5 febrero 2016]. Disponible en: https://www.ilo.org/global/aboutthe-ilo/mission-and-objectives/lang--es/index.htm

5. Ochoa E, Madoz, A. Consumo de alcohol y otras drogas en el medio laboral. Med Segur Trab. [Internet] 2008; 54(213):25-32. [Acceso 2 feb 2016]. Disponible en: http://scielo.isciii.es/pdf/mesetra/v54n213/original1.pdf 6. Cheng WJ, Huang MC, Cheng $\mathrm{Y}$, Chen $\mathrm{CH}$, Chen $\mathrm{CJ}$. Consumption of Alcoholic Energy Drinks Is Associated with Work-related Injury or Disease Among Manual Workers in Taiwan. Alcohol Alcohol. [Internet] 2015; 50(4): 458-62. [Acceso 2 feb 2016]. doi.org/10.1093/alcalc/agx106
7. Foster SA, Dissanaike SD. Prevalence and consequences of positive blood alcohol levels among patients injured at work. J Emerg Trauma Shock. [Internet] 2014; 7:268-73. [Acceso 2 Feb 2016]. doi: [10.4103/0974-2700.142748]

8. Marques $\mathrm{PH}$, Jesus $\mathrm{V}$, Olea SA., Vairinhos $\mathrm{V}$, Jacinto $C$. The effect of alcohol and drug testing at the workplace on individual's occupational accident risk. Safety Sci. [Internet] 2014; 68:108-12. [cited Feb 32016]. Available from: https://research.unl.pt/ files/368190/2014_Marques\%20et\%20al_SS_68_ effect\%20of\%20alcohol\%20and\%20drug\%20testing.pdf 9. Kifle M, Engdaw D, Alemu K, Sharma HR, Amsalu S, Feleke $A$, et al. Work related injuries and associated risk factors among iron and Steel industries workers in Addis Ababa, Ethiopia. Occup Med Health Affairs. [Internet] 2014; 63:211-6. [cited Feb 32016]. Available from: doi. org/10.1016/j.ssci.2013.11.020

10. Dzeng RJ, Wang SH, Fang YC. Quantitative evaluation of the impact of night shifts and alcohol consumption on construction tiling quality. Applied Ergonom. [Internet] 2015;50:223-36. [cited Feb 2016]. Available from: doi. org/10.1016/j.apergo.2015.03.008

11. González R, Hidalgo G, Salazar JG. Preciado M.L. Instrumento para medir la calidad de vida en el trabajo. México: Instituto de investigación en salud ocupacional; 2009. 59 p.

12. Bronfenbrenner $U$. The ecology of human development. Massachussets: Harvard University Press; 1979. $321 \mathrm{p}$.

13. Sallis JF, Owen N, Fisher EB. Ecological models of health behavior. In: Glanz K, Rimer BK, Viswanath K. Health behavior and health education:theory, research and practice. New York: Jossey-Bass/Wiley; 2008. 533 p. 14. Walker LO, Avant KC. Strategies for theory construction in nursing. 5th ed. Pearson; 2011. 243 p. utilización de los materiales sujetos a la licencia. 